\title{
Vortex Dynamics Analysis of Straight-Body- Type-Fuselage Fighter Using CFD Simulation
}

\author{
Sutrisno Sutrisno ${ }^{1, *}$, Deendarlianto Deendarlianto", Tri Agung Rohmat¹, Setyawan Bekti Wibowo¹, Sigit Iswahyudi \\ Sutrisno S (iD https://orcid.org/0000-0001-5161-7607 \\ How to cite \\ Deendarlianto D (D) https: / / orcid.org/0000-0002-1242-2951 \\ Sutrisno; Deendarlianto; Rohmat TA; Wibowo SB; Iswahyudi \\ $S$ (2020) Vortex Dynamics Analysis of Straight -Body-Type- \\ Fuselage Fighter Using CFD Simulation J Aerosp Tecnol Manag, \\ Rohmat TA (D) https://orcid.org/0000-0001-5335-0272 \\ 12: e1020. https://doi.org/10.5028/jatm.v12.1104 \\ Wibowo SB (D) https: / / orcid.org/0000-0002-3067-1660
}

Iswahyudi S (D) https://orcid.org/0000-0002-9478-4074

\begin{abstract}
The choice for using a fighter fuselage in a fighter jet design affects a vortex generation advantageous in maneuverability. To study the effect of straight-body-type-fuselage [SBTF] on the vortex dynamic, a computational fluid dynamics (CFD) method is used, in order to simulate a model of SBTF fighter. The simulation uses Q-criterion to probe vortices, and a logarithmic grid to emphasize the micro-gridding effect of the turbulent boundary layer. The results show detailed quantitative velocity, pressure, trajectory of the vortex core, and wing negative surface pressure distribution (SPD), providing clear pictures of opportunity for performance improvement, better lift, agility, and maneuverability of a fighter if a model requires a new design.
\end{abstract}

KEYWORDS: Vortex dynamic analysis; Vortex core; Q-criterion; Flight capability; Rolled-up vortex.

\section{INTRODUCTION}

The development of aircraft fighter began with a progress in research on delta wing, that persists until now. Vortex structures developed on the double-delta wings have shown the vortex flows on the leading-edges of the wing, and on the canard. Both vortex structures interact with each other (Abderrahmane 2013). Computational modeling of vortex breakdown on a delta wing, with a technique developed based on the use of jet-flaps, is capable of capturing the vortex breakdown location for a variety of jet exit angles (Vlahostergios et al. 2013). Numerical and experimental investigations of a non-slender delta wing have examined the leading-edge vortex flap effect (Cai et al. 2014). In-depth research about design (Bitencourt et al. 2011), control, the interaction between systems, and measuring tools continues to press forward. Chen et al. (2013) have investigated a set behavior of a large number of vortices in the plane (Chen et al. 2013; Hövelmann 2016). Up to then, scientists have investigated the significance of the rolled-up vortex $(\mathrm{RuV})$ effect, in order to exploit more practicality and benefit from the vortex-dynamic mechanism.

Some research related to interconnected aerodynamics mechanisms has focused on 3D separation and its control (Manolesos 2013). An experimental investigation has found the flow mechanisms responsible for the aircraft loss of static directional stability (Jing et al. 2016). The method of three-dimensional measurements of vortex breakdown has focused more on vortex dynamics mechanism (Calderon et al. 2012). Medford (2012) has investigated the aerodynamics of a maneuvering unmanned aircraft vehicle (UAV) model and its control through leading-edge curvature change. Scientists have examined flight dynamics and control modeling of damaged asymmetric aircraft (Ogunwa and Abdullah 2016; Bacon and Gregory 2018; Nguyen et al. 2006; Zhang et al. 2018; Asadi et al. 2014).

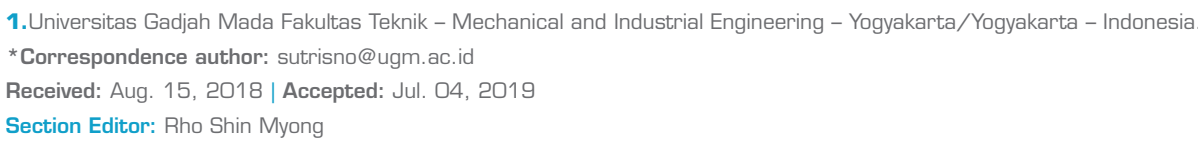


Further study for future aircraft design, including for military purposes, could develop important advancement of aircraft technology. Flight physical aspects and methods of future military aircraft designs have been thoroughly explored (Hitzel 2015). Schminder (2012) has carried out a feasibility study of different methods for the use in aircraft conceptual design. Zhang et al. (2013) have investigated numerically the characteristics between canard and wing of canard-forward swept wing aircraft configurations at different canard positions, focusing on the interference between canard and wing. Several numerical model simulations of F-16XL fighters have been conducted. Geometry and computational grids with structured grids, and the unstructured grids used in cranked-arrow wing aerodynamics international project have been executed. Numerical simulation of flight-test conditions using delayed detached-eddy simulation has been performed. Simulations at flight conditions using hybrid near-body/off body CFD have been carried out as well (Boelens et al. 2009; Lofthouse and Cummings 2017; Morton et al. 2017). Nevertheless, the application of the Q-criterion algorithm so far has not been combined with the footprint of the streamline for the vortex development, in order to scrutinize the complete picture of the vortex dynamics. Due to fuselage effect, fighters fuselage configurations give different spreading effect on the fighter wing, determining the produced lift force.

Following to the fuselage effect theory, canard fighters have two different fighter types, namely: a) the SBTF fighter; and b) the bird's body type fuselage (BBTF) fighter (named after the fact that the head and the neck of the fighters resemble the ones of a bird). SBTF fighters include Rafale, Eurofighter Typhoon, Chengdu J-10, and SAAB Gripen.

The field data (available at https://aviatia.net/rafale-vs-eurofighter/) shows that Rafale and Eurofighter are well known to have very high dog-fighting abilities. However, there are still more opportunities to strengthen their agility and maneuverability, which, in principle, means increasing their lift coefficients at high AoA (angle of attack). This requires a deep research on the fighter vortex dynamic, which will determine the role of RuV effect, as well as the role of vortex cores in generating negative SPD on the right surface and reducing losses of the lift coefficient of the fighter. In this case, the role of $Q$-criterion is very important.

Several eminent scientists have explored numerical investigation to some fighters. Boelens (2012) have conducted CFD analysis of the flow around the X-31 aircraft at a high AoA. Chen et al. (2016) have studied the effect of sideslip on high-AoA vortex flow over a close-coupled canard configuration. Ghoreyshi et al. (2016) have performed a simulation validation of static and forced motion flow physics of a canard configured TransCruiser. Ghoreyshi et al. (2013) have learned transonic aerodynamic load modeling of X-31 aircraft pitching motions. Schütte and Rein (2007) have examined experimental and numerical aspects of simulating unsteady flows around the X-31 configuration. In this paper, the Q-criterion algorithm is employed, so that the footprint of the streamline for the vortex development and the wall shear are combined to scrutinize the complete picture of the vortex dynamics.

Vorticities have to be illustrated precisely. In order to distinguish between vortical structures and shear flows, an additional vortex detection algorithm, the Q-criterion, has been put to use (Holmén 2012). A Q-criterion denotes that vortices of an incompressible flow are identified as connected fluid regions, with a positive second invariant of the velocity-gradient tensor $(\nabla u)$. Then, $\nabla u=S+\Omega$ is decomposed into symmetrical and antisymmetrical parts - being $S=0.5\left(\nabla u+\nabla u^{\mathrm{T}}\right)$ the strain-rate tensor, $\Omega=0.5\left(\nabla u-\nabla u^{\mathrm{T}}\right)$ the vorticity tensor, and $T$ the transpose. This defines the rate of strain and vorticity tensors, respectively. If $Q=0.5\left(\|\Omega\|_{\mathrm{E}}{ }^{2}-\|S\|_{\mathrm{E}}{ }^{2}\right)>0,\|.\|_{\mathrm{E}}$ represents the Euclidean norm. Holmén (2012) also described other similar vortex detection algorithms, namely: delta criterion, lambda-2 criterion, swirling strength criterion, and enhanced swirling strength criterion.

Görtz (2005), based on several in-depth, systematical investigation, summarized that: i) vortex breakdown in a flow over delta wings is practically an inviscid phenomenon. In that flow, the Euler and Navier-Stokes equations would predict the same vortex breakdown location, where ii) the nonlinear interaction between a forebody vortex and a burst wing leading-edge vortex can be predicted using the Navier-Stokes equations. Then, iii) the computations of vortical flows, especially the vortex breakdown, are highly sensitive to local grid resolution. It was also found that iv) the detached-eddy simulations (DES) were shown to be promising for time-accurate viscous computations of separated flows in general, and vortex breakdown. It was identified that v) the vortical flows at high incidence are inherently unsteady and require time-accurate computations, especially for predicting the breakdown location.

The purpose of this paper is to analyze aircraft fighter designs that employ structured logarithmic mesh, applying, in the second section, numerical simulation methodology to maintain the accuracy of the turbulent boundary layer on the Kolmogorov 
microscale. In general, dog-fighting techniques are carried out by flying the fighter at slow speed, short range, and high AoA. Therefore, this simulation applies $0.3 \mathrm{M}$ for the fighter speed, where $\mathrm{M}$ is the Mach number.

Simulation and measurement results display visualizations of vortex cores and wall shear streamlines that show negative SPD. The negative pressure areas of the vertical wall close to the canard and the wing leading-edge, including the canard and wing vortex core that stay away from the wing surface, both reduce the fighter lift capability. Those cases waste much energy. Several pieces of evidence on the wing reveal its concluding results, specially the negative SPD on the wing that exhibits the agility and maneuverability of the fighter. Subsequently, the results are extended to discuss some improvement opportunity for it to be the best fighter with excellent agility and maneuverability.

\section{METHODOLOGY}

The model studied in this research is a SBTF fighter-like model, as shown in Fig. 1, with some simplifications in the symmetrical models, and some detailed drawings, such as an antenna. SBTF fighters include Rafale, Eurofighter Typhoon, SAAB Gripen, and Chengdu. In this study, the authors chose one of these fighters as a representative of the SBTF. A net on the SBTF fighter aircraft was created by identifying the aircraft parts and then dividing them into several blocks, based on surface changes of the aircraft. The hexahedral mesh nets were structured by changing the net size, starting from the wall portion, as the smallest size, and enlarging logarithmically to the outside (Boelens 2012).

To obtain the optimal cell number, first, it was necessary to conduct mesh independence test, as shown in Table 1 . The previous test, with 5 million cells, had achieved the convergence of the coefficient values of lifting force. In this modeling case, the number of created cells was 6,012,908 ( $\sim 6$ million). To determine the smallest size of the net on the wall, the value of $y+$ (dimensionless wall distance) equals 4 , with the lowest cell value of $0.017 \mathrm{~mm}$. These results are in accordance with reference about mesh independence study on aircraft that have previously been carried out (Wibowo 2019).

Computational domains are made so large that the computational boundaries do not interfere with the flow of the aircraft model. The set equation model used is

$$
\partial / \partial \mathrm{t}(\rho u)+\nabla \cdot(\rho u \otimes u+\nabla p)=\nabla \cdot \tau+\rho g
$$

where $\rho$ is the density, $u$ is the flow velocity, $\nabla$ is the divergence, $p$ is the pressure, $\tau$ is the deviatoric stress tensor of the order of two, and $g$ is body accelerations acting on the continuum.

In order to solve the equation set, the finite volume method could be used. The discretization model of pressure-velocity coupling uses PISO (Pressure-Implicit with Splitting of Operators); convection term uses QUICK (Quadratic Upwind Interpolation for Convective Kinematics); and pressure term uses a second-order scheme.

Since the dog-fighting is carried out at slow speed, the fighter simulation is conducted at a speed of $0.3 \mathrm{M}$. The vortex identification criterion is valid for incompressible flows only, as, apparently, also is the Q-criterion. Incompressible flow is the one at a Mach number $\mathrm{M} \leq 0.3$. When such number assumes different, higher values in the maneuver space, it causes drag divergence, whose Mach number is the one at which the aerodynamic drag on an airfoil begins to increase rapidly as the Mach number continues to increase. The substantial increase in drag is caused by the formation of a shock wave on the upper surface of the airfoil, which can induce flow separation and adverse pressure gradients on the aft portion of the wing.

Therefore, the vortex dynamics pattern around the fighter would be symmetrical, causing, to save time, the computation to be done with the half model. Figure 2 shows the shape of the net and gridding above the canard. The computational domain was box-shaped with half the model that would be formed being symmetrically reflected. The boundary conditions in the computational domain were determined, including the inlet (or velocity inlet), the outlet (pressure outlet), and the symmetrical planes. 


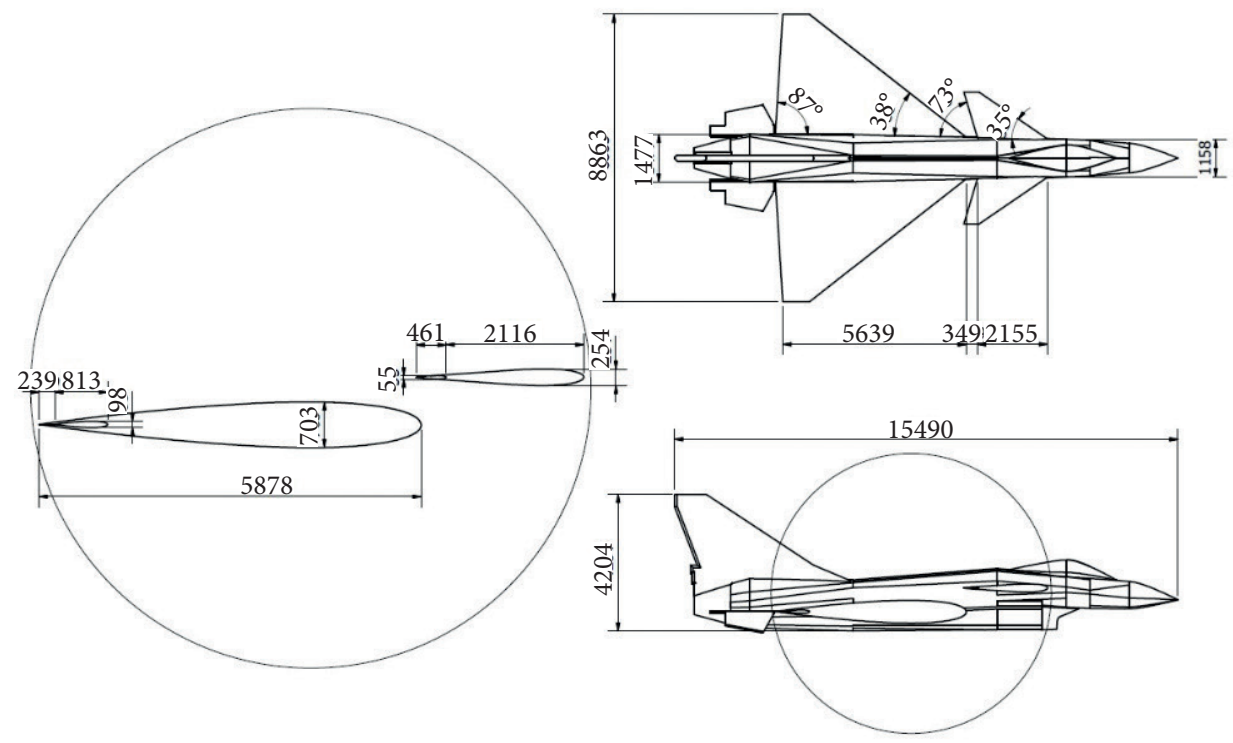

Figure 1. Dimension of the SBTF fighter-like CFD model.

Table 1. Mesh independent test for different cell number.

\begin{tabular}{|c|c|c|c|}
\hline Criteria & AoA & $\boldsymbol{C}_{\boldsymbol{l}}$ & Error \\
\hline Boelens 2012 & $30^{\circ}$ & 1.02157 & $5.14 \%$ \\
\hline 1.3 million grids & $30^{\circ}$ & 1.074078 & $2.01 \%$ \\
\hline 3.1 million grids & $30^{\circ}$ & 1.042085 & $0.44 \%$ \\
\hline 5.2 million grids & $30^{\circ}$ & 1.026022 & \\
\hline
\end{tabular}

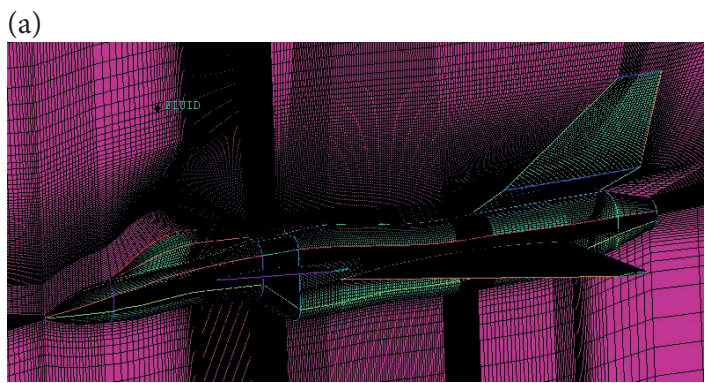

(b)

(c)
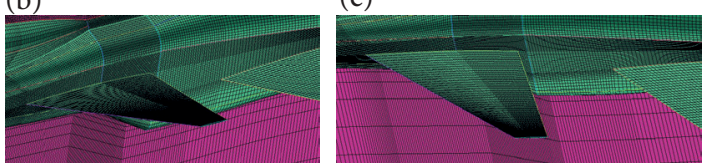

Figure 2. Picture of the grid on the symmetry plane for the SBTF fighter CFD model with fuselage configuration, showing computational domain for (a) the half model (symmetrical), (b) detailed gridding above the canard, and c) detailed gridding above main wing leading-edge and above the canard.

This study involved several variations of the AoA, ranging from $20^{\circ}$ to $70^{\circ}$. The flow rate was set at an inlet velocity of $0.3 \mathrm{M}$ $(114 \mathrm{~m} / \mathrm{s})$ flowing on the surface of the plane with a $0.08 \%$ turbulence intensity. The flow analysis, based on the Navier-Stokes equation, employed the finite volume method. Vortex dynamics analysis was used to analyze the fuselage and RuV effects of the 
SBTF-like fighter canard. Said type of analysis involves flow visualization (which consists of steps to plot the limiting streamline, drawing footprints from time to time), in order to analyze fighters, and a review of the measurement results.

The flow visualization plot of the main vortex center is also presented, which may also generate the second vortex center. Afterwards, the measurement results are analyzed. The first result is related to the strength of the vortex center, which is the velocity of the axial vortex center relative to the local free stream velocity $U c / U \infty(z)$ and the pressure coefficient $(C p)$ of the vortex center. We then measure the surface pressure distributions at the left and the right fighter wing surfaces. The trajectory height and the spanwise location of the vortex center are then measured. To determine the magnitude of the vortex core, the $Q$-criterion is used with the equation:

$$
Q=\frac{1}{2}\left[|\Omega|^{2}-|S|^{2}\right]>0
$$

In this study, $Q$-criterion flow identification was employed where vortices of an incompressible flow were identified as connected fluid regions with a positive second invariant of the velocity-gradient tensor $\nabla u$. Being $\nabla u=S+\Omega, S$ is the rate-to-strain tensor; and $\Omega$ is the vorticity tensor. $Q=0.5\left(\|\Omega\|_{\mathrm{E}}{ }^{2}-\|S\|_{\mathrm{E}}^{2}\right)>0$. The symbol $\|.\|_{\mathrm{E}}$ represents the Euclidean norm.

\section{PHYSICS AND OPERATION OF WATER TUNNEL}

Flow visualization in low-speed water tunnels has been an effective method for the analysis of complex flow fields. Although this type of test is conducted at very low Reynolds numbers, the vortices and their generation, interaction, and breakdown in water tunnel are relatively insensitive to Reynolds number. Erickson (1981) concluded that flow fields that are vortex-dominated are similar regardless of Reynolds number. The flow field surrounding the SBTF configuration at high AoA can be characterized as vortex-dominated. Therefore, Erickson's findings provide confidence that the results from the water tunnel test are reliable. In this experiment, the SBTF lift coefficient $\left(C_{\mathrm{L}}\right)$ and drag coefficient $\left(C_{\mathrm{D}}\right)$ measurements in water tunnel were used to validate the accuracy of CFD modeling. The CFD simulation gave a satisfying result.

Gama Water tunnel storage can accommodate 1,200 liters of water, connected via a silencer tank, a contraction cone to the test section, with a $200 \mathrm{~mm} \times 200 \mathrm{~mm}$ cross section. The silencer, or tranquilizer, tanks contain fibers and nets to stabilize the flow as it passes through the test section. As shown in Fig. 3, water flows through two honeycombs to allow it to flow laminar. The Gama Water Tunnel research has been conducted by Wibowo et al. (2018), who examined the relationship between the aerodynamic forces and vortex-dynamic phenomena in delta aircraft models as the primary aerodynamic mechanism of fighter aircraft. The results are compared to various studies on the same model in several means, such as wind tunnel, real scale aircraft, and water tunnel models from other studies. The good compatibility between the qualitative results $\left(C_{1}-C_{\mathrm{d}}\right)$ and the quantitative output (vortex structure formation) gives an indication that the extensive capability of water tunnel facilities could be applied to analyze comprehensively the aerodynamic character of aircraft (Wibowo et al. 2018).

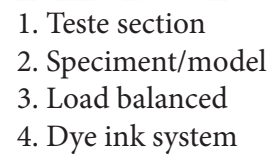

1. Teste section

2. Speciment/model

3. Load balanced

4. Dye ink system

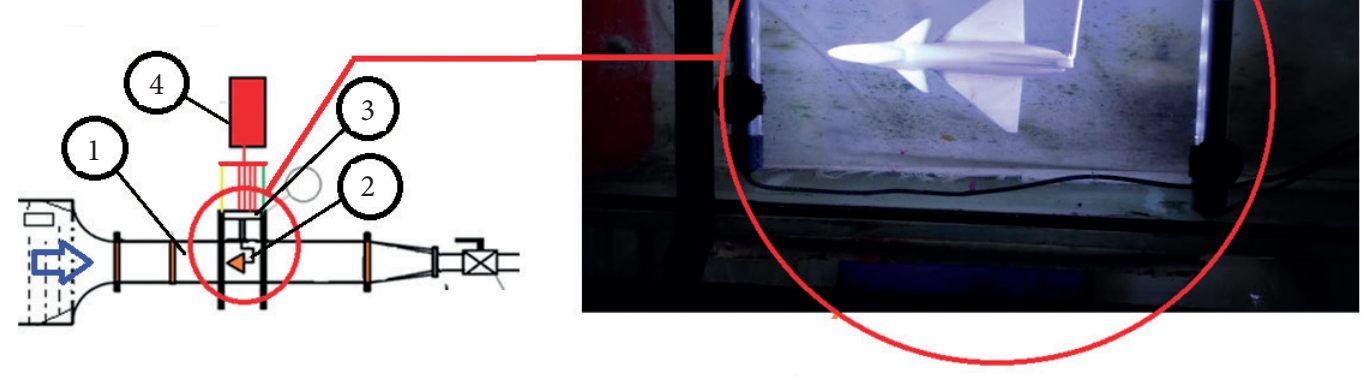

Figure 3. Water tunnel facility with 12,000 liters of water storage with water speed $1 \mathrm{~m} / \mathrm{s}$ at the $20 \mathrm{~cm} \times 20 \mathrm{~cm}$ test section. 


\section{RESULTS AND DISCUSSIONS}

\section{NUMERICAL VALIDATION}

The wind tunnel experimental data, used for validation, is a result of an X-31 experiment with high AoA aircraft configuration. The model data, used at first by Boelens ( 2012), is from the DNW Low-Speed Wind Tunnel at Braunschweig, DLR. This test run constitutes AoA $(\alpha)$ ranging from $-60^{\circ}$ to $55^{\circ}$. The wind tunnel velocity has been $60 \mathrm{~m} / \mathrm{s}$, which corresponds to a Mach number of 0.18 .

Figure 4 shows the comparison between this experimental data against the lift coefficient $C_{\mathrm{L}}$ and drag coefficient $C_{\mathrm{D}}$ for X-31 CFD model, employing the $k-\omega, k-\varepsilon$, and shear stress transport (SST) turbulence equations, where the $k$ is the turbulence kinetic energy, the $\omega$ is the specific rate of dissipation, and the $\varepsilon$ is the rate of dissipation of turbulent kinetic energy. The calculation results of the Navier-Stoke equation, utilizing ANSYS Fluent' software, applied to X-31 CFD models, were compared to the reference experiment. It shows that the model with the SST equation gives the best match in the comparison. Wibowo et al. (2018) recommends using a turbulent model SST or $k$ - $\omega$, that is suitable for analyzing the vortex phenomenon in fighter models with low computational loads. For that reason, this study used the SST turbulence model to get good results in the simulation.
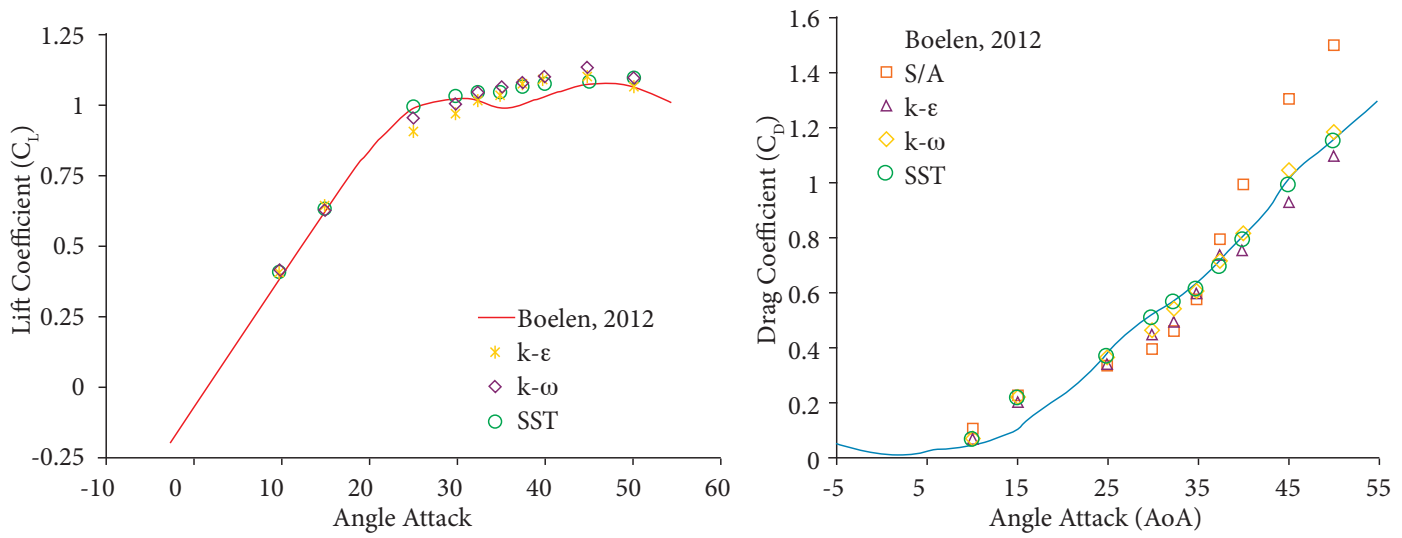

Figure 4. Lift coefficient $\left[C_{L}\right]$ and drag coefficient $\left[C_{D}\right]$ for X-31 CFD model employing $k-\omega, k-\varepsilon$, and SST turbulence equations, compared to the reference experiment (Boelens 2012) for different $A \circ A(\alpha)=0^{\circ}$ to $60^{\circ}$.

\section{WATER TUNNEL TEST VALIDATION}

The $C_{\mathrm{L}}$ and $C_{\mathrm{D}}$ of SBTF CFD model employing the SST turbulence equations were validated against the result of SBTF water tunnel experiment for different $\operatorname{AoA}(\alpha)=0^{\circ}$ to $60^{\circ}$, as shown in Fig. 5 . The comparison of $C_{\mathrm{L}}$ indicates a similarity with the standard deviation $<3 \%$.
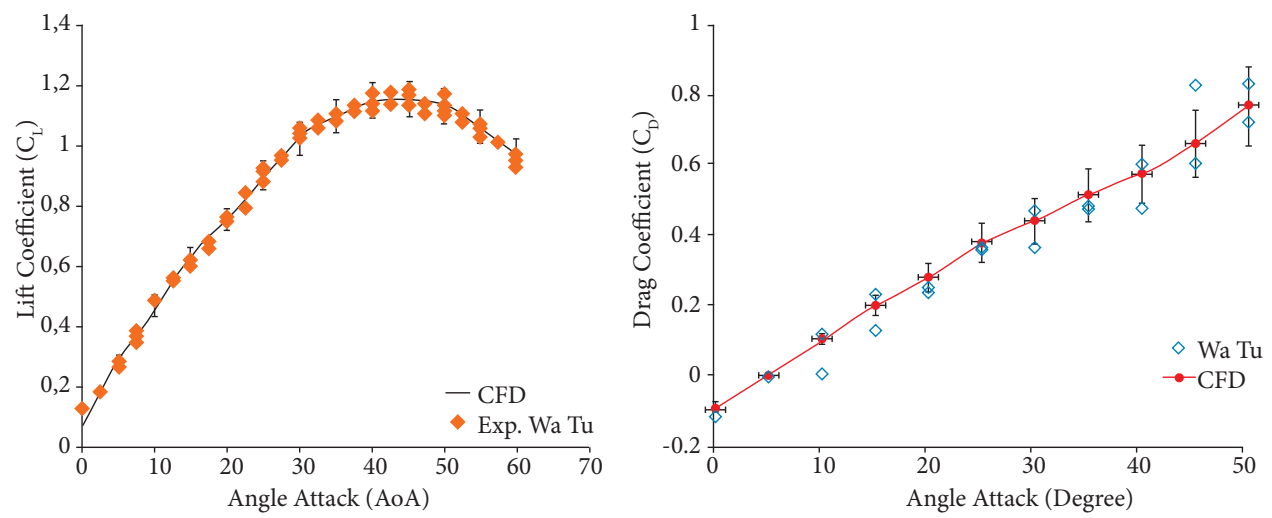

Figure 5. Lift coefficient $\left(C_{L}\right)$ and drag coefficient $\left(C_{D}\right)$ of SBTF CFD model employing the SST turbulence equations compared to the result of SBTF water tunnel experiment (Exp. WaTu) for different $A \circ A[\alpha]=0^{\circ}$ to $60^{\circ}$. 


\section{VORTEX CORE AND WALL SHEAR STREAMLINES FOR THE FIGHTER MODEL}

Following the fuselage effect theory, in Figs. $6 \mathrm{a}$ to $6 \mathrm{~d}$, it is possible to observe the wing vortex core leading to the stall occurrence. They denote their vortex cores at different $\operatorname{AoA}(\alpha)=20^{\circ}, 40^{\circ}, 60^{\circ}$, and $70^{\circ}$. Figure 7 shows the flow visualization of canard and wing vortex cores (which designates their vortex breakdowns), and the flow configuration of the wall pressure. The colored circles denote the vortex strength. White lines present the trajectory of the vortex cores through the circles. As the fighter moves forward, the fuselage deflects the canard flow, so the curved head contour cleaves the flow apart. It strengthens the RuV effects above the canard. As the AoA increases, the vortex core strengthens, thereby generating canard lift. Figure 7 likewise displays the trajectory of vortex core at various different AoA $(\alpha)=20^{\circ}$ to $70^{\circ}$, and presents as well the adverse SPD at the wing and the vertical walls, showing prime vortex reattachment. Figures $7 \mathrm{c}$ and $7 \mathrm{~d}$ also demonstrate the "negative" SPD at the vertical wall close to the canard and the wing. Regrettably, it would waste a significant amount of energy, probably due to the less implementation of the blendedwing body design during planning.

Figure 8 illustrates the streamlines, wall-shear streamlines, and total pressure loss around the canard. The canard deflects wing vortex-core streamlines. The wing vortex center breaks into two vortices, namely: a vortex tip as it is at the wing tip; and asecond vortex, which is suspected to be a RuV effect as it is in the middle of the wing, which is shown in Figs. 8c and 8d. Figure 9 explains more detailedly and schematically than Fig. 8 why canard location and canard deflection could affect jet deflection from the wing and make the vortex move away from the wing surface. Therefore, we need to examine the vortex jet from the vortex center, due to the fuselage-head/canopy-wing configuration effects. It determines the impact from the canard vortex core jet into the wing, associated with the fuselage and canard-wing interaction. The vortex centers effect could be spreading, shrinking, and widening upwards or downwards.
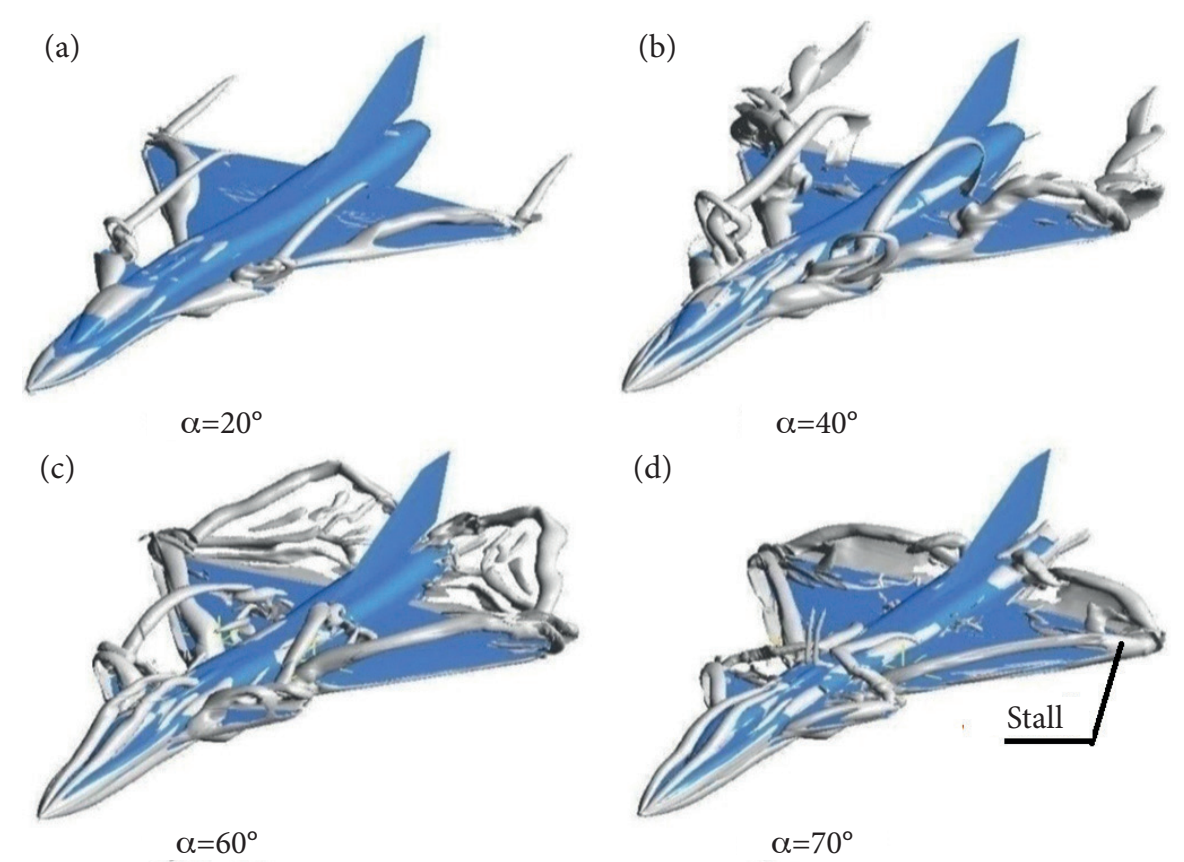

Figure 6. Vortex flow dynamics around head, canard, and wing denote their vortex cores at different $A \circ A(\alpha)=20^{\circ}, 40^{\circ}, 60^{\circ}$, and $70^{\circ}$, using Q-criterion $=3.6 \times 10^{-5} \mathrm{~s}^{-2}$. 
(a)

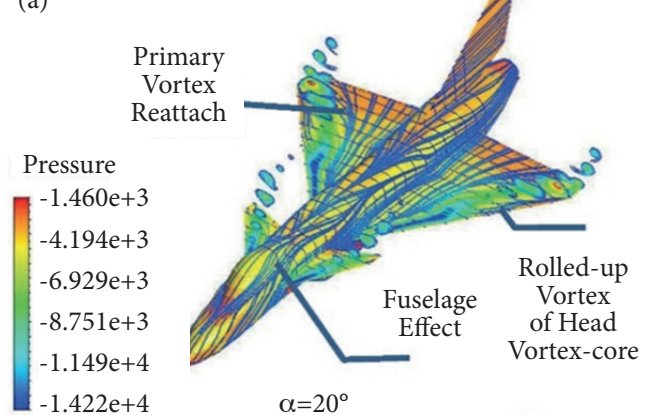

$[\mathrm{Pa}]$

(c)

a

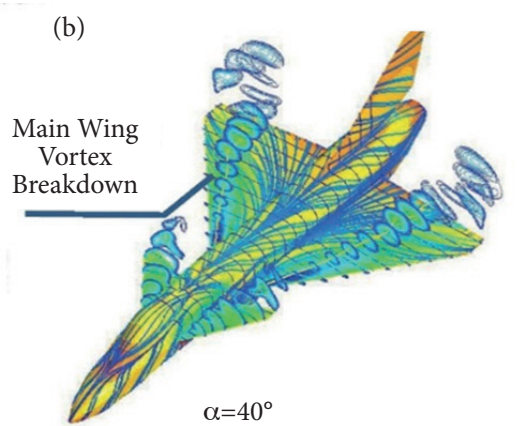

(d)

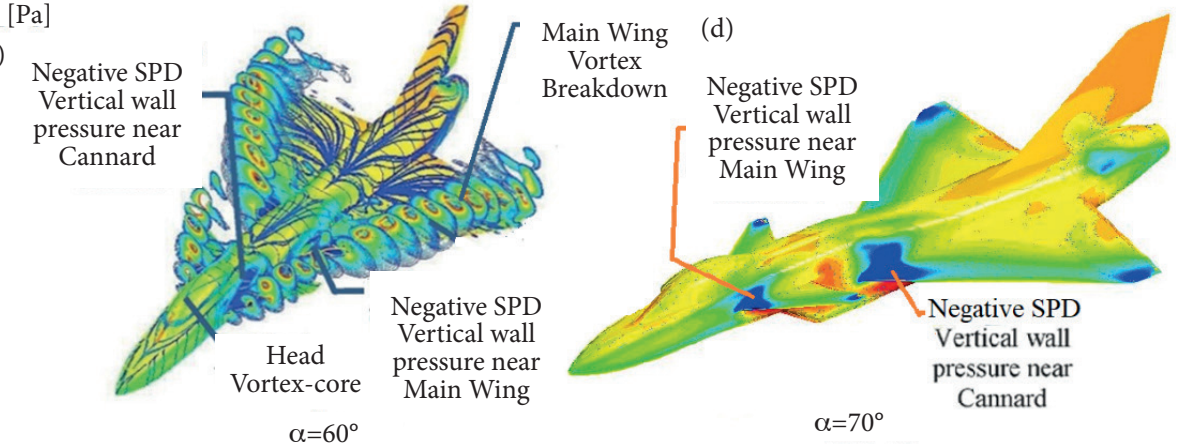

Figure 7. Flow configuration of wall pressure, vortex centers, and vortex cores at the canard, wing, and vertical wall, denoted by wall-shear streamlines and total pressure loss. It shows primary vortex reattachment, and the trajectory of the vortex core at different $A \circ A[\alpha]=20^{\circ}, 30^{\circ}, 60^{\circ}$, and $70^{\circ}$.

(a)

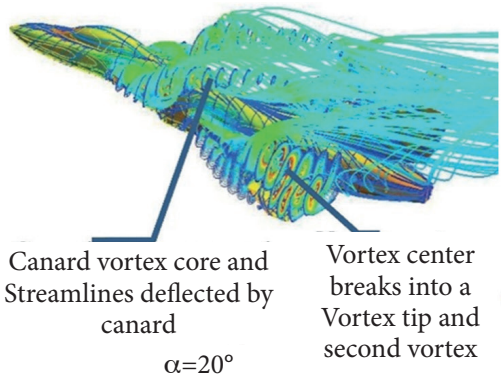

(c)

Pressure

$-1.460 \mathrm{e}+3$

$-4.194 \mathrm{e}+3$

$-6.929 e+3$

$-8.751 e+3$

$-1.149 \mathrm{e}+4$

$-1.422 \mathrm{e}+4$

$[\mathrm{Pa}]$

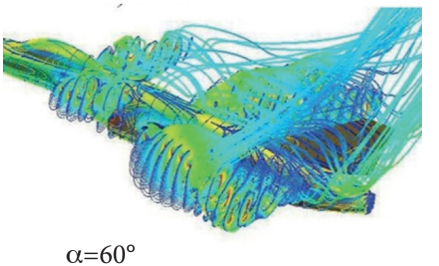

(b)

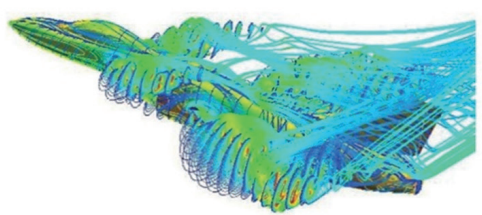

$\alpha=50^{\circ}$

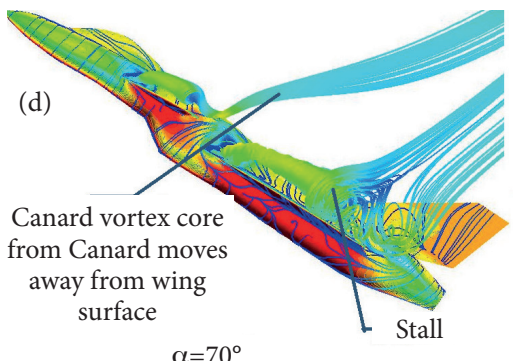

Figure 8. Flow visualization shows the wall-shear streamlines and total pressure loss around the canard and the wing vortex cores. 


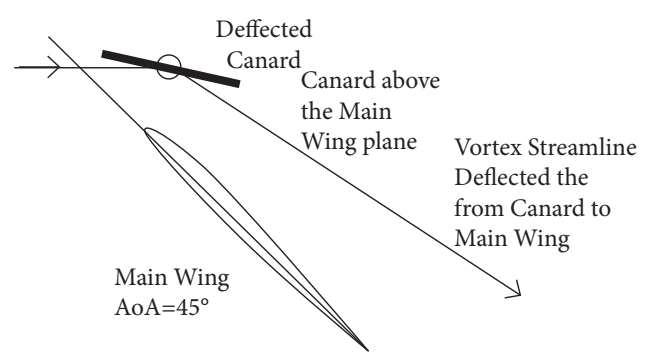

b) the main wing have lower height

Figure 9. Effect of canard location and deflection. The wing line at a lower height than the deflected canard axis could deflect the vortex core farther away from the wing surface.

\section{MAIN WING VORTEX CORE TRAJECTORY MEASUREMENT}

The center of the vortex core location above the wing surface is determined by slicing into pieces longitudinally (plane $A_{x y}$, where $X$ is the vertical axis and $Y$ is the spanwise one), from the leading-edge to the tail, and finding the location of the $2 \mathrm{D}$ highest axial speed, point by point, along the vortex core line in plane $A_{\mathrm{xy}}(z, \alpha)$, where $A_{\mathrm{xy}}=\left(x_{\mathrm{Uc}}(z), y_{\mathrm{Uc}}(z), z, \alpha\right)$, being $z$ the longitudinal axis; and $U c$ the axial canard vortex center velocity $(\mathrm{m} / \mathrm{s})$. The height of the vortex core trajectory is $x_{U c}(z, \alpha)$, and the spanwise of the vortex core trajectory is $y_{\mathrm{Uc}}(z, \alpha)$. $U c(z)$ is the axial wing vortex core velocity, and $U c / U \infty(z)$ is the ratio of the axial wing vortex core velocity relative to the free stream velocity. The negative $C p_{\text {surface }}(z)$ is the negative SPD at (a) $30 \%$, and (b) $60 \%$ wing root chords.

Figures 10 to 12 reveal the vortex core dynamics, showing the vortex core strength and its trajectory to produce the wing negative SPD, which, by its turn, generates the lift force of the fighter, with its detail vortex dynamic mechanism. Figure 10a demonstrates the velocity ratio variation $U c / U \infty(z)$, namely: the ratio of the axial wing vortex core velocity relative to the free stream velocity. The wing vortex core strength at $\mathrm{AoA}$ from $10^{\circ}$ to $20^{\circ}$ is high in the front section, which extends to the rear. Furthermore, at AoA from $30^{\circ}$ to $60^{\circ}$, the cores are potent in the front and the rear sections, but have a weak center in the middle. Also, at AoA from $70^{\circ}$ to $80^{\circ}$, the vortex centers are weak almost in the entire sections, in the direction of the stall stage. Figure $9 \mathrm{~b}$ shows the pressure coefficient negative $C p(z)$ variation along the wing vortex core at AoA from $10^{\circ}$ to $80^{\circ}$. It illustrates that, on AoA from $10^{\circ}$ to $20^{\circ}$, the vortex core has a sufficiently strong axial vortex strength, yet they nevertheless produce weak negative pressure in the core. Eventually, the pressure strengthens at AoA from $30^{\circ}$ to $40^{\circ}$; the negative $C p$ in the core reaches the highest at AoA from $50^{\circ}$ to $60^{\circ}$; and then weakens again at AoA from $70^{\circ}$ to $80^{\circ}$.

In Fig. 11a, the negative SPD at $30 \%$ of the chords at the AoA of $10^{\circ}$ is very weak, and the negative $C p$ at the surface is between 1 and 6. At AoA from $30^{\circ}$ to $50^{\circ}$, the negative $C p$ is between 3 and 10, reaching the highest at $30^{\circ}$, and then weakening again at AoA from $70^{\circ}$ to $80^{\circ}$. In Fig. $11 \mathrm{~b}$, with the negative SPD at $60 \%$ of the chord at $10^{\circ}$, the negative $C P$ is between 1 and 4 ; and at AoA from $30^{\circ}$ to $50^{\circ}$, the negative $C p$ reaches the highest only between 2 and 5 at $40^{\circ}$, weakening again at AoA from $70^{\circ}$ to $80^{\circ}$. Figures $12 \mathrm{a}$ and $12 \mathrm{~b}$ show that the trajectory height of vortex cores and the spanwise location along the length of the wing are far from the surface of the wing.
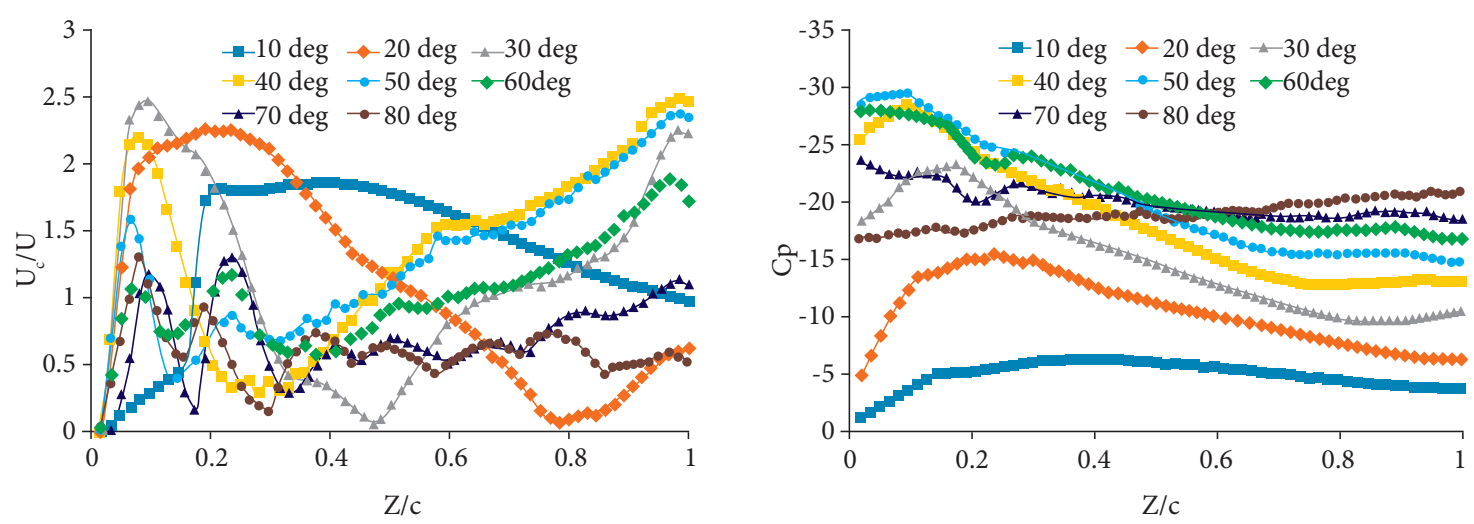

Figure 10. (a) Velocity ratio variation $U_{c} / U_{\infty}[z]$ along the wing vortex core; (b) negative $C p(z)$ of curves along the wing vortex core at different $A \circ A(\alpha)=10^{\circ}$ to $80^{\circ}$. 

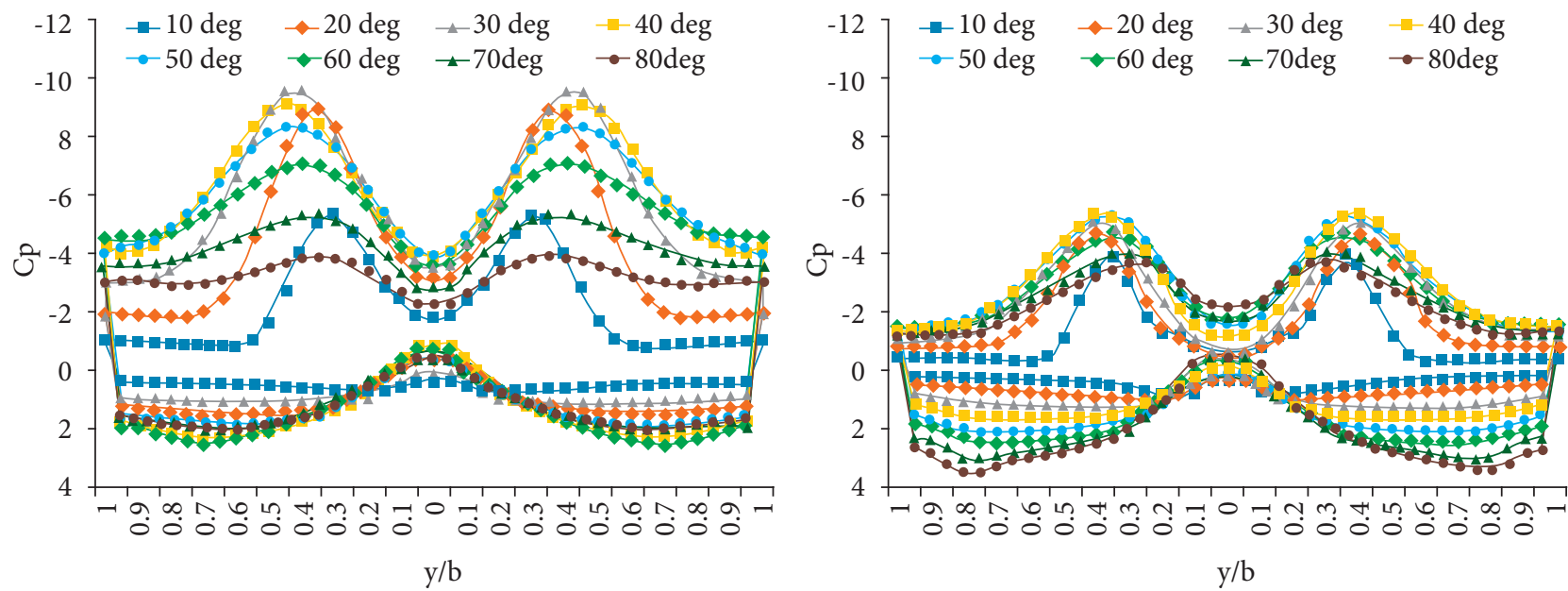

Figure 11. Surface pressure distributions at (a) $30 \%$, and (b) $60 \%$ wing root chord at different $A \circ A(\alpha)=10^{\circ}$ to $80^{\circ}$.
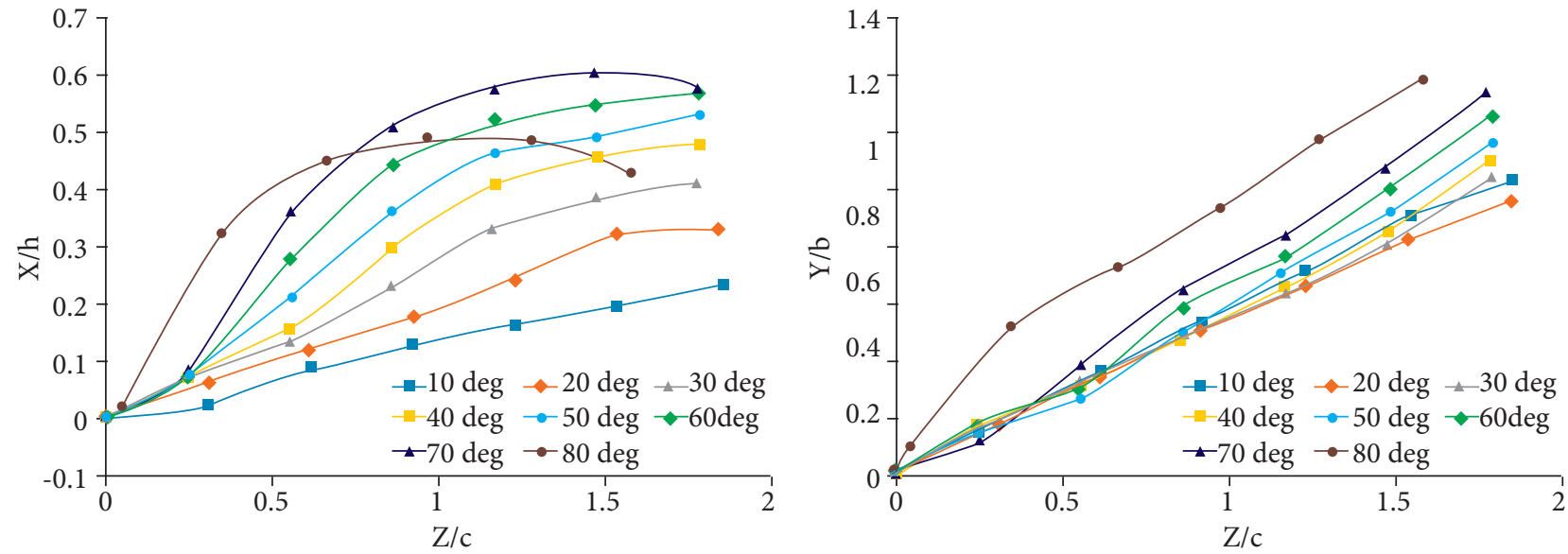

Figure 12. (a) Height of vortex core; (b) spanwise location along the length of the wing vortex core.

Even though this model is remarkably simplified, without a flap, movable canard, or movable elevon, it might be suggested that wing vortex cores for AoA from $70^{\circ}$ to $80^{\circ}$ need to be reinforced to remove the weakness. The negative SPD at $60 \%$ of the chord is relatively weak almost at the entire AoA. Thus, the SPD needs better distribution. The advantages and the result of implementing Q-criterion to probe vortices are, namely, that the strength could be measured, and the direction of the vortices region could be specified precisely. The advantages of using a logarithmic grid to emphasize the micro-gridding effect of the turbulent boundary layer are that the number of cells could be minimized and still the result could be optimized by setting the cell size to match the Kolmogorov microscale in order to catch effectively the turbulent boundary layer effect. Therefore, the advantages of using Q-criterion to probe vortex regions are that it would be possible to specify the detailed quantitative trajectory of the vortex core, as well as the development of velocity, pressure, and wing negative SPD could be explained. Furthermore, it would be possible to explain performance improvement, better lift, agility, and maneuverability of the aircraft.

\section{PERFORMANCE IMPROVEMENT OPPORTUNITY OF SBTF-LIKE FIGHTER MODEL}

Rafale and Eurofighter are well-known to have very high dog-fighting abilities (available at https://aviatia.net/rafale-vseurofighter/). However, there are still plenty of opportunities to strengthen their agility and maneuverability, which, in principle, is increasing their lift coefficients at high AoA. As shown in Table 2, it could be put together several possibilities for additional 
changes, in order to generate $\mathrm{RuV}$ effects to canard vortex cores and breakdowns, negative SPD, vortex core trajectory heights (VCTH), fighter high AoA capability (HAC), and a reduced stall tendency. The movable flap would homogenize the vortex core distribution over the wing, and the movable elevon, whether inboard or outboard, would improve the pressure distribution in the wing.

Table 2. Vortex dynamics benefits and disadvantages, due to additional constituents, devices, and little fuselage configuration design change.

\begin{tabular}{|c|c|c|}
\hline $\begin{array}{c}\text { Constituents and devices } \\
\text { in general }\end{array}$ & Benefit & Disadvantage \\
\hline Blended wing body design & Based on flow visualization analysis \\
\hline Canard location and deflection & $\begin{array}{c}\text { Enhanced lift-to-drag ratio, and } \\
\text { weight and lift distribution; } \\
\text { homogeneous stress distribution } \\
\text { Improve the spreading effect of the jet }\end{array}$ & $\begin{array}{c}\text { Requires high technology } \\
\text { metallurgy and composite }\end{array}$ \\
\hline & Based on the vortex core trajectory analysis \\
\hline
\end{tabular}

Based on the vortex core trajectory analysis

Deflected canard (Fig. 9)

Tube and wing design of fighter aircraft

Backside with similar flat heights from head to tail

Different height canard from the wing (see Fig. 9)

Single inlet (under the body)

Movable flap

Movable elevon
Lower canard VCTH,

reduced wing stall tendency, increased fighter HAC and better SPD

Middle manufacturing technology, shorter design timeline

Metallurgy and composite needs high technology

Shorter design timeline

\section{Based on logical analysis}

Open rooms to the canard flow

Middle technology

Fewer bumps effect

Better RuV distribution over the wing

Better controllable negative SPD

in the wing rear part
Waste energy, negative SPD on the vertical wall close to the canard and the wing

Less obstructed negative SPD

Reduce fighter HAC

Several constituents, devices, and design changes might be required.

- Based on flow visualization analysis: a deflected canard could be used to lower the canard VCTH, in order to improve performance, as shown in Fig. 9. The implementation of a blended wing body design would enhance lift-to-drag ratio, and weight/lift distribution, and homogenize stress distribution. However, this approach requires advanced technology in metallurgy and composites.

- Based on the vortex core trajectory analysis: a deflected canard (Fig. 9) would lower canard VCTH, reduce wing stall tendency increased fighter HAC, and gave better SPD. A different canard height from the wing (Fig. 9) would reduce fighter HAC.

- Based on logical analysis: Additional constituents would include double engines to reduce the effect of bumps on the backside of the fighter. A double air-inlet provides limited room for canards beside the cockpit canopy. Canards are arranged beside the turbine inlet or hang down beside the inlets. 


\section{CONCLUSION}

This report demonstrates the flow visualization of the fuselage and RuV effects on aircraft, as well as the trajectory measurement of the canard and the wing vortex cores of a canard fighter. The use of the Q-criterion algorithm, combined with the footprint of the vortex development and streamlines, enables us to derive a complete picture of the vortex dynamics mechanism.

The negative SPD on the vertical walls, near the canard and the wing, strengthens as AoA increases, due to less implementation of blended wing body design, thereby wasting a significant amount of energy. The trajectory of the vortex cores gradually moves away from the wing surface as $\alpha$ increases, reducing, then, the $C_{\mathrm{L}}$.

The strength of the axial vortex velocity of the fighter model is dynamically affected by its canard and wing vortex cores. At AoA of $30^{\circ}, 40^{\circ}$, and $50^{\circ}$, the system forms the vortex core, which has a potent axial vortex velocity at the front and the rear of the wing, that is weak in the middle. Moreover, the negative CP along the wing core is almost evenly distributed, and reaches the maximum at $\alpha=40^{\circ}$. At 30\% of the wing chord, the negative SPD is still potent as $\alpha$ increases from $30^{\circ}$ to $40^{\circ}$, and then weakens. However, at $60 \%$ of the wing chord, all negative SPD at the wing surface is entirely weak. Moreover, at $\mathrm{AoA}$ from $70^{\circ}$ to $80^{\circ}, \mathrm{Uc} / \mathrm{U}^{\circ}$, negative $C p$ along the vortex core, and the negative SPD at 30 and $60 \%$ of the wing chord are weak, as the fighter model stalls.

Recommended performance, and agility and maneuverability improvements include a combination of the system with the constituents and devices of different styles. Modifications, such as a blended wing body design, and an alteration from single to double engines, in order to deliver more power and to reduce the effect of bumps, can be made to improve performance, HAC, and SPD.

\section{AUTHOR'S CONTRIBUTION}

Conceptualization, Sutrisno; Wibowo SB; Iswahyudi S; Data Curation, Wibowo SB; Iswahyudi S; Methodology, Wibowo SB; Formal Analysis, Sutrisno; Wibowo SB; Iswahyudi S; Resources, Iswahyudi S; Validation, Wibowo SB; Project Administration, Sutrisno; Investigation, Rohmat TA; Software, Rohmat TA; Wibowo SB; Iswahyudi S; Visualization, Rohmat TA; Wibowo SB; Writing - Original Draft, Sutrisno; Deendarlianto; Rohmat TA; Wibowo SB; Iswahyudi S; Writing - Review \& Editing, Sutrisno; Deendarlianto; Wibowo SB; Iswahyudi S; Funding Acquisition, Sutrisno; Resources; Supervision, Sutrisno; Deendarlianto; Rohmat TA.

\section{REFERENCES}

Abderrahmane A (2013)Visualisation of vortex structures developed on the upper surface of double-delta wings. J Aeronaut Aerosp Eng 2(4):4-7. doi: https://doi.org/10.4172/2168-9792.1000118

Asadi D, Sabzehparvar M, Atkins EM, Talebi HA (2014) Damaged airplane trajectory planning based on flight envelope and motion primitives. J Aircr 51(6):1740-1757. doi: https://doi.org/10.2514/1.C032422

Bacon BJ, Gregory IM (2018) General equations of motion for a damaged asymmetric aircraft. NASA Langley Research Center, Hampton, VA, 23681

Bitencourt LO, Pogorzelski G, de Freitas RM, Azevedo JLF (2011) A CFD-based analysis of the 14-Bis aircraft aerodynamics and stability. J Aerosp Technol Manag 3(2):137-146. doi: https://doi.org/10.5028/jatm.2011.03021711

Boelens OJ (2012) CFD analysis of the flow around the X-31 aircraft at high angle of attack. Aerosp Sci Technol 20(1):38-51. doi: https://doi.org/10.1016/j.ast.2012.03.003

Boelens OJ et al. (2009) F16-XL geometry and computational grids used in cranked-arrow wing aerodynamics project international. J Aircr 46(2):369-376. doi: https://doi.org/10.2514/1.34852

Cai J, Pan S, Li W, Zhang Z (2014) Numerical and experimental investigations of a nonslender delta wing with leading-edge vortex flap. Comput Fluids 1-17. doi:10.1016/j.compfluid.2014.03.011 
Calderon DE, Wang Z, Gursul I (2012) Three-dimensional measurements of vortex breakdown. Exp Fluids 53:293-299. doi: https://doi. org/10.1007/s00348-012-1317-1

Chen M, Liu P, Guo H, Qu Q (2016) Effect of sideslip on high-angle-of-attack vortex flow over close-coupled canard configuration. J Aircr 53(1):217-230. doi: https://doi.org/10.2514/1.C033305

Chen Y, Kolokolnikov T, Zhirov D (2013) Collective behaviour of large number of vortices in the plane. Proceedings of the Royal Society A: Mathematical, Physical and Engineering Science 469:1-12.doi: https://dx.doi.org/10.1098/rspa.2013.0085

Erickson GE (1981) Vortex flow correlation. Tech Rep AFWAL-TR (ADA108725). OHIO: Flight Dynamics Laboratory, Air Force Wright Aeronautical Laboratories; [accessed 122017 Nov]. http://www.dtic.mil/docs/citations/ADA108725

Ghoreyshi M, Cummings RM, Ronch A Da, Badcock KJ (2013) Transonic Aerodynamic load modeling of X-31 aircraft pitching motions. AIAAJ 51(10):2447-2464. doi: https://doi.org/10.2514/1.J052309

Ghoreyshi M, Korkis-Kanaan R, Jirasek A, Cummings RM, Lofthouse AJ (2016) Simulation validation of static and forced motion flow physics of a canard configured TransCruiser. Aerosp Sci Technol 48:158-177. doi: https://doi.org10.1016/j.ast.2015.11.008

Görtz S (2005) Realistic simulations of delta wing aerodynamics using novel CFD methods (PhD thesis). Stockholm: Royal Institute of Technology.

Hitzel SM (2015) Flightphysical aspects and methods of future military aircraft designs. Paper presented 5th Challenges in European Aerospace CEAS Air \& Space Conference 1-25; Delft, Netherlands.

Holmén V (2012) Methods for vortex identification. Lund University; [accessed 2017 Jul 12]. http://lup.lub.lu.se/student-papers/ record/3241710

Hövelmann AN (2016) Analysis and control of partly-developed leading-edge vortices (PhD thesis). Munich: Technischen Universität München.

Jing W, Yankui W, Xueying D (2016) An experimental investigation on static directional stability. Chinese J Aeronaut 29(6):1527-1540. doi: https://doi.org/10.1016/j.cja.2016.10.008

Lofthouse AJ, Cummings RM (2017) Numerical simulations of the F-16XL at flight-test conditions using delayed detached-eddy simulation. J Aircr 1-23. doi: https://doi.org/10.2514/1.C034045

Manolesos M (2013) Experimental and computational study of three-dimensional separation and its control using passive vortex generators (PhD thesis). Athens: National Technical University of Athens.

Medford CM (2012) The aerodynamics of a maneuvering UCAV 1303 aircraft model and its control through leading-edge curvature change (MS Thesis). California: Naval Postgraduate School.

Morton SA, Mcdaniel DR, Air E, Base F (2017) F-16XL simulations at flight conditions using hybrid near-body/offbody computational fluid dynamics. J Aircr 54(6):2050-2069. doi: https://doi.org/10.2514/1.C034095

Nguyen N, Krishnakumar K, Kaneshige J, Nespeca P (2006) Dynamics and adaptive control for stability recovery of damaged asymmetric aircraft. Paper presented AIAA Guidance, Navigation, and Control Conference and Exhibit; Colorado, USA. doi: https://doi. org/10.2514/6.2006-6049

Ogunwa T, Abdullah EJ (2016) Flight dynamics and control modelling of damaged asymmetric aircraft. Paper presented IOP Conference Series: Materials Science and Engineering 152(1):012022. doi: https;//doi.org/10.1088/1757-899X/152/1/012022

Schminder JPW (2012) Feasibility study of different methods for the use in aircraft conceptual design (MS thesis). Linköping: Linköping University.

Schütte A, Rein M (2007) Experimental and numerical aspects of simulating unsteady flows around the X-31 configuration. Paper presented 3rd International Symposium on Integrating CFD and Experiments in Aerodynamics. Proceedings of the Institution of Mechanical Engineers Part G Journal of Aerospace Engineering 223(4):309-321; Colorado, USA. doi: https://doi.org/10.1243/09544100JAER0387

Vlahostergios Z, Missirlis D, Yakinthos K, Goulas A (2013) Computational modeling of vortex breakdown control on a delta wing. Int J Heat Fluid Flow 39:64-77. doi: https;//doi.org/10.1016/j.jijheatfluidflow.2012.12.002

Wibowo SB, Sutrisno, Rohmat TA et al. (2018) An investigation into the use of GAMA water tunnel for visualization of vortex breakdown on the delta wing. Paper presented 9th International Conference on Thermofluids. AIP Conference Proceedings, p. 050007; Yogyakarta, Indonesia. doi: https://doi.org/10.1063/1.5049998

Wibowo SB, Sutrisno, Rohmat TA (2018) An evaluation of turbulence model for vortex breakdown detection over delta wing. Arch Mech Eng LXV(3):399-415. doi: https://doi.org/10.24425/124489

Wibowo SB, Sutrisno, Rohmat TA (2019) Study of mesh independence on the computational model of the roll-up vortex phenomena on fighter and delta wing model. Int J Fluid Mech Res 46(5):427-439. doi: https://doi.org/10.1615/InterJFluidMechRes.2018025530

Zhang GQ, Yu SCM, Chien A, Angeles L, Yang SX (2013) Aerodynamic characteristics of canard-forward swept wing aircraft configurations. J Aircr 50(2):378-387. doi: https://doi.org/10.2514/1.C031740

Zhang S, Shuang W, Meng $Q$ (2018) Control surface faults neural adaptive compensation control for tailless flying wing aircraft with uncertainties. Int J Control Autom Syst 16(4):1660-1669. doi: https://doi.org/10.1007/s12555-017-0454-y 